\title{
К ЗАДАЧЕ ДИНАМИЧЕСКОГО ВОССТАНОВЛЕНИЯ ВОЗМУЩЕНИЯ ПРИ ДЕФИЦИТЕ ИНФОРМАЦИИ
}

\author{
В. Л. Розенберг
}

\begin{abstract}
Задача восстановления неизвестного внешнего воздействия в системе линейных обыкновенных дифференциальных уравнений исследуется с позиций подхода теории динамического обращения. Рассматривается постановка, в которой реконструкция возмущения проводится синхронно с развитием процесса на основе неточной дискретной информации о части координат фазовой траектории. Предлагается конечношаговый программно реализуемый алгоритм решения, основанный на методе вспомогательных позиционно управляемых моделей, получена оценка его точности. Новизна статьи состоит в рассмотрении постановки обратной задачи для динамической системы, в которой возмущение, подлежащее реконструкции, стеснено геометрическими ограничениями и не входит в измеряемую компоненту.
\end{abstract}

Ключевые слова: система обыкновенных дифференциальных уравнений, дефицит информации, динамическое восстановление, управляемая модель.

V. L. Rozenberg. On a problem of dynamic reconstruction under incomplete information.

The problem of reconstructing an unknown external influence in a system of linear ordinary differential equations is investigated on the basis of the approach of the theory of dynamic inversion. A statement is considered in which the disturbance is reconstructed synchronously with the process from incomplete discrete information on a part of coordinates of the phase trajectory. A finite-step software-oriented solution algorithm based on the method of auxiliary closed-loop models is proposed, and its error is estimated. The novelty of the paper is that we consider the inverse problem for a dynamic system in which the disturbance to be reconstructed is subject to geometric constraints and is not included in the measured component.

Keywords: system of ordinary differential equations, incomplete information, dynamic reconstruction, controlled model.

MSC: 49K15, 93C41

DOI: $10.21538 / 0134-4889-2019-25-1-207-218$

\section{Введение}

Задачи восстановления (реконструкции) неизвестных параметров управляемых систем в режиме реального времени на основе неполной и/или неточной информации о фазовом состоянии вкладываются в проблематику обратных задач динамики; как правило, они являются некорректными (в силу неединственности искомой характеристики и/или разрывности обратного оператора) и требуют применения регуляризирующих процедур. Настоящая статья ориентирована на подход, разработанный А. В. Кряжимским, Ю. С. Осиповым и их коллегами (см. [1-3] и библиографию в них) и получивший название метода динамического обращения. Он основан на сочетании принципов теории позиционного управления [4] и идей теории некорректных задач [5]. Фактически задача реконструкции сводится к задаче управления по принципу обратной связи вспомогательной динамической системой (моделью). Управление в модели адаптируется к результатам текущих наблюдений таким образом, что его реализация во времени устойчиво приближает (в подходящем смысле) неизвестный вход.

Метод динамического обращения был реализован для широкого круга задач, описываемых обыкновенными дифференциальными уравнениями (ОДУ), дифференциально-функциональными уравнениями, уравнениями и вариационными неравенствами с распределенными параметрами, а также стохастическими дифференциальными уравнениями. При этом восстанавливались различные характеристики систем, а именно неизвестные возмущения, начальные и граничные данные, коэффициенты дифференциальных операторов и т. д. Были созданы 
устойчивые алгоритмы, работающие для некоторых классов частично наблюдаемых систем. В случае конечномерной системы роль входного сигнала могли играть измерения части координат фазового вектора, а в случае бесконечномерной системы - значения решения на некоторых подмножествах области определения. Такие задачи были сформулированы и решены, например, в [3;6;7].

В данной статье исследуется задача построения конструктивного алгоритма динамического восстановления неизвестного возмущения для частично наблюдаемой системы ОДУ специального вида.

\section{1. Постановка задачи}

Рассматривается система линейных ОДУ следующего вида:

$$
\begin{aligned}
& \dot{y}(t)=A y(t)+B z(t)+f_{0}(t), \quad \dot{z}(t)=C z(t)+D y(t)+E u(t)+f_{1}(t), \\
& t \in T=\left[t_{0}, \vartheta\right], y\left(t_{0}\right)=y_{0}, z\left(t_{0}\right)=z_{0} .
\end{aligned}
$$

Здесь $(y(\cdot), z(\cdot))$ - фазовая траектория системы, $y(t)=y\left(t ; t_{0}, y_{0}, z_{0}, u(\cdot)\right) \in \mathbb{R}^{n_{1}}, z(t)=$ $z\left(t ; t_{0}, y_{0}, z_{0}, u(\cdot)\right) \in \mathbb{R}^{n_{2}} ; u(\cdot)$ - возмущение, принимающее значения из заданного выпуклого компакта $P \in \mathbb{R}^{m}$ и имеющее ограниченную на $T$ вариацию, $U(\cdot)=\left\{u(\cdot) \in V\left(T ; \mathbb{R}^{m}\right)\right.$ : $u(t) \in P \quad \forall t \in T\} ; A, B, C, D$ и $E-$ постоянные матрицы размерности $n_{1} \times n_{1}, n_{1} \times n_{2}$, $n_{2} \times n_{2}, n_{2} \times n_{1}$ и $n_{2} \times m$ соответственно; векторные функции $f_{0}(\cdot)$ и $f_{1}(\cdot)$ размерности $n_{1} \times 1$ и $n_{2} \times 1$ суммируемы на $T$ с квадратом нормы. Решение задачи Коши понимается в смысле Каратеодори, предполагается абсолютная непрерывность траектории $(y(\cdot), z(\cdot))$.

Обсуждаемая задача состоит в следующем. Неизвестное возмущение $u(t)$, действующее на систему (1.1), входит только во второе уравнение. В то же время в дискретные, достаточно частые моменты времени $\tau_{i} \in T, \tau_{i}=t_{0}+i \delta, \delta=\left(\vartheta-t_{0}\right) / l, i \in[0: l]$, поступает неточная информация о первой фазовой компоненте $y\left(\tau_{i}\right)$. Результаты измерений, векторы $\xi_{i} \in \mathbb{R}^{n_{1}}$, удовлетворяют неравенствам

$$
\left\|\xi_{i}-y\left(\tau_{i}\right)\right\|_{n_{1}} \leq h,
$$

где $h \in(0,1)$ - величина погрешности измерений. В дальнейшем символы $\|\cdot\|_{n}$ и $(\cdot, \cdot)_{n}$ означают соответственно евклидову норму и скалярное произведение в пространстве $\mathbb{R}^{n}$.

Требуется указать алгоритм динамического восстановления неизвестной функции $u(t)$ по информации $\xi_{i}, i \in[0: l]$, причем отклонение приближения от искомого входа в метрике пространства $L_{2}\left(T ; \mathbb{R}^{m}\right)$ должно быть сколь угодно мало при достаточно малом $h$ и специальным образом согласованном с ним шаге временной дискретизации $\delta=\delta(h)$.

В настоящей статье алгоритм решения описанной выше задачи конструируется в рамках теории динамического обращения. В связи с неполнотой информации (а именно с возможностью измерения в моменты $\tau_{i}$ не всего фазового состояния системы $\left(y\left(\tau_{i}\right), z\left(\tau_{i}\right)\right)$, а лишь его части $\left.y\left(\tau_{i}\right)\right)$, в модель вводится блок динамической аппроксимации неизвестной координаты $z(t)$, который играет роль поставщика информации о текущем полном фазовом состоянии системы. Эта информация оперативно передается на блок, формирующий по закону обратной связи модельное управление, приближающее реальное возмущение.

Новизна работы состоит в рассмотрении постановки обратной задачи для динамической системы, в которой возмущение, подлежащее реконструкции, стеснено геометрическими ограничениями и не входит в измеряемую компоненту. Изучение обратных задач динамики в условиях дефицита информации было начато со случая, когда неизвестное возмущение присутствует в измеряемой компоненте системы (см. [6]). Разработанный алгоритм использовал возможность формального разрешения уравнения, описывающего наблюдаемую часть фазового состояния, относительно возмущения и предполагал построение на этой основе подходящей модели, восстанавливающей в режиме реального времени как неизмеряемую компоненту, так и неизвестную функцию. Методика получила дальнейшее развитие; были рассмотрены задачи 
реконструкции такого типа для частично наблюдаемых систем ОДУ в различных постановках (см. обзор [7]). В работе [8] решена задача гарантированного управления системой ОДУ при поступлении информации о части фазового состояния, помеха в данном случае входила в ненаблюдаемую компоненту. Постановка, аналогичная рассматриваемой в указанной статье, для случая отсутствия геометрических ограничений на неизвестное возмущение исследована в [9]. Модификации некоторых конструкций из [8;9] использованы при доказательстве основного результата.

\section{2. Алгоритм решения задачи}

Фундаментальные матрицы систем $\dot{y}(t)=A y(t)$ и $\dot{z}(t)=C z(t)$ обозначим соответственно символами $Y(t)=\exp (A t)$ и $Z(t)=\exp (C t)$. Заметим, что в силу свойств матричной экспоненты можно указать такие константы $\omega$ и $\hat{\omega}$, что выполняются неравенства

$$
\|Y(t)\|_{n_{1}}^{2} \leq \exp (\omega t), \quad\|Z(t)\|_{n_{2}}^{2} \leq \exp (\hat{\omega} t) .
$$

Потребуем выполнения следующих условий, стесняющих правую часть системы (1.1).

У с л о в и е 1 . Пусть $n_{2} \leq n_{1}$ и существует число $d_{*}>0$ такое, что неравенство $\|B v\|_{n_{1}} \geq$ $d_{*}\|v\|_{n_{2}}$ выполняется при всех $v \in \mathbb{R}^{n_{2}}$.

У с л о в и е 2 . Пусть $m \leq n_{2}$ и существует число $\hat{d}_{*}>0$ такое, что неравенство $\|E u\|_{n_{2}} \geq$ $\hat{d}_{*}\|u\|_{m}$ выполняется при всех $u \in \mathbb{R}^{m}$.

Отметим, что условия 1, 2 справедливы, например, для квадратных невырожденных матриц $B, E$.

Введем систему-модель, которая состоит из двух блоков и имеет следующий вид:

$$
\begin{aligned}
& \dot{w}_{y}^{h}(t)=A w_{y}^{h}(t)+B v^{h}(t)+f_{0}(t), \quad \dot{w}_{z}^{h}(t)=C w_{z}^{h}(t)+D w_{y}^{h}(t)+E u^{h}(t)+f_{1}(t), \\
& t \in T=\left[t_{0}, \vartheta\right], w_{y}^{h}\left(t_{0}\right)=y_{0}, w_{z}^{h}\left(t_{0}\right)=z_{0} .
\end{aligned}
$$

Здесь $w_{y}^{h}(t) \in \mathbb{R}^{n_{1}}, w_{z}^{h}(t) \in \mathbb{R}^{n_{2}}$ - модельные переменные; $v^{h}(t) \in \mathbb{R}^{n_{2}}, u^{h}(t) \in \mathbb{R}^{m}$ - модельные управления. Идея решения такова. Первый блок, идентификатор, используя неточные измерения вида (1.2), формирует управление $v^{h}(\cdot)$, аппроксимирующее неизмеряемую координату $z(\cdot)$ в метрике пространства $L_{2}\left(T ; \mathbb{R}^{n_{2}}\right)$. Второй блок, контроллер, на основе полученной информации о полном фазовом состоянии системы (1.1) вычисляет управление $u^{h}(\cdot)$, приближающее искомое возмущение $u(\cdot)$ в метрике пространства $L_{2}\left(T ; \mathbb{R}^{m}\right)$. Оба управления, подающиеся на вход модели, вырабатываются в соответствии с законами обратной связи.

Запишем дискретный аналог модели (2.2):

$$
\begin{aligned}
& w_{y}^{h}\left(\tau_{i+1}\right)=w_{y}^{h}\left(\tau_{i}\right)+\left(A w_{y}^{h}\left(\tau_{i}\right)+B v^{h}\left(\tau_{i}\right)+f_{0}\left(\tau_{i}\right)\right) \delta, w_{y}^{h}\left(t_{0}\right)=y_{0}, \\
& w_{z}^{h}\left(\tau_{i+1}\right)=w_{z}^{h}\left(\tau_{i}\right)+\left(C w_{z}^{h}\left(\tau_{i}\right)+D w_{y}^{h}\left(\tau_{i}\right)+E u^{h}\left(\tau_{i}\right)+f_{1}\left(\tau_{i}\right)\right) \delta, w_{z}^{h}\left(t_{0}\right)=z_{0} .
\end{aligned}
$$

Сначала выберем функции $\alpha(h), \hat{\alpha}(h):(0,1) \rightarrow \mathbb{R}^{+}$(регуляризаторы) и семейство разбиений $\Delta_{h}, h \in(0,1)$, отрезка $T$ со свойствами

$$
\begin{gathered}
\Delta_{h}=\left\{\tau_{i}\right\}_{i=0}^{l}, \quad \tau_{i}=\tau_{h, i}, \quad l=l_{h}, \quad \tau_{i+1}=\tau_{i}+\delta, \quad \delta=\delta(h), \\
\delta(h) \rightarrow 0, \quad \alpha(h) \rightarrow 0, \quad \hat{\alpha}(h) \rightarrow 0 \quad \text { при } \quad h \rightarrow 0, \\
\rho(h)=(h+\delta(h)+\alpha(h))^{1 / 2}+(h+\delta(h)) \alpha^{-1}(h), \quad \rho(h) \rightarrow 0, \\
\hat{\rho}(h)=\left((\rho(h))^{1 / 2}+\hat{\alpha}(h)\right)^{1 / 2}+(\rho(h))^{1 / 2} \hat{\alpha}^{-1}(h), \quad \hat{\rho}(h) \rightarrow 0 .
\end{gathered}
$$

Процесс управления моделью организуем следующим образом. В начальный момент времени $t_{0}$ фиксируем $h, \Delta_{h}, \alpha(h)$ и $\hat{\alpha}(h)$. Работу алгоритма разобьем на $l$ однотипных шагов. 
На $i$-м шаге, который выполняется на интервале $\left(\tau_{i}, \tau_{i+1}\right], i \in[0:(l-1)]$, исходными данными для вычислений служат измерение $\xi_{i}$ и сформированное к моменту $\tau_{i}$ состояние модели $w_{y}^{h}\left(\tau_{i}\right)$, $w_{z}^{h}\left(\tau_{i}\right)$. Выполняются следующие операции. Сначала блок-идентификатор по измерению $\xi_{i}$ и значению компоненты $w_{y}^{h}\left(\tau_{i}\right)$ вычисляет вектор

$$
v_{i}^{h}=v_{i}^{h}\left(\xi_{i}, w_{y}^{h}\left(\tau_{i}\right)\right)=\arg \min \left\{L_{v}\left(\tau_{i}, \alpha, v\right): v \in V(d)\right\},
$$

где

$$
\begin{gathered}
L_{v}\left(\tau_{i}, \alpha, v\right)=2 \exp \left(-\omega \tau_{i+1}\right)\left(\left(w_{y}^{h}\left(\tau_{i}\right)-\xi_{i}\right), B v\right)_{n_{1}}+\alpha(h)\|v\|_{n_{2}}^{2}, \\
d=\sup \left\{\left\|z\left(t ; t_{0}, y_{0}, z_{0}, u(\cdot)\right)\right\|_{n_{2}}: t \in T, u(\cdot) \in U(\cdot)\right\}, \\
V(d)=\left\{v \in \mathbb{R}^{n_{2}}:\|v\|_{n_{2}} \leq d\right\} .
\end{gathered}
$$

Существование числа $d$ очевидно. Затем блок-контроллер на основе аппроксимации $v_{i}^{h}$ и значения компоненты $w_{z}^{h}\left(\tau_{i}\right)$ определяет вектор

$$
u_{i}^{h}=u_{i}^{h}\left(v_{i}^{h}, w_{z}^{h}\left(\tau_{i}\right)\right)=\arg \min \left\{L_{u}\left(\tau_{i}, \hat{\alpha}, u\right): u \in P\right\},
$$

где

$$
L_{u}\left(\tau_{i}, \hat{\alpha}, u\right)=2 \exp \left(-\hat{\omega} \tau_{i+1}\right)\left(\left(w_{z}^{h}\left(\tau_{i}\right)-v_{i}^{h}\right), E u\right)_{n_{2}}+\hat{\alpha}(h)\|u\|_{m}^{2} .
$$

После этого согласно формулам (2.3) модель переходит в состояние $w_{y}^{h}\left(\tau_{i+1}\right), w_{z}^{h}\left(\tau_{i+1}\right)$. Фактически в течение промежутка времени $\left(\tau_{i}, \tau_{i+1}\right]$ на вход системы $(2.2)$ подаются два постоянных управления

$$
v^{h}(t)=v_{i}^{h}, \quad u^{h}(t)=u_{i}^{h},
$$

тем самым формируются кусочно-постоянные функции $v^{h}(t), u^{h}(t), t \in T$. На следующем, $(i+1)$-м шаге, выполняются аналогичные действия. Работа алгоритма заканчивается в конечный момент времени $t=\vartheta$. Сформулируем основной результат статьи.

Теорема. Пусть выполняются условия согласования параметров (2.4). Тогда для модельного управления $u^{h}(\cdot)$, вычисляемого согласно (2.7)-(2.9), имеет место следующая оценка качества аппроксимачии:

$$
\left\|u^{h}(\cdot)-u(\cdot)\right\|_{L_{2}\left(T ; \mathbb{R}^{m}\right)}^{2} \leq C_{1} \hat{\rho}(h),
$$

где $C_{1}$ - некоторая константа, не зависящая от очениваемых величин.

Д о к а з а т е л ь с т в о. Вначале покажем, что процедура построения функции $v^{h}(\cdot)$ согласно $(2.5),(2.6),(2.9)$ обеспечивает аппроксимацию неизмеряемой компоненты $z(\cdot)$, реализовавшейся в системе (1.1), в следующем смысле:

$$
\left\|v^{h}(\cdot)-z(\cdot)\right\|_{L_{2}\left(T ; \mathbb{R}^{\left.n_{2}\right)}\right.}^{2} \leq C_{2} \rho(h),
$$

где $C_{2}$ - некоторая константа, не зависящая от оцениваемых величин.

Введем функционал типа Ляпунова и оценим его изменение:

$$
\varepsilon_{h}(t)=\exp (-\omega t)\left\|w_{y}^{h}(t)-y(t)\right\|_{n_{1}}^{2}+\alpha(h) \int_{t_{0}}^{t}\left(\left\|v^{h}(\tau)\right\|_{n_{2}}^{2}-\|z(\tau)\|_{n_{2}}^{2}\right) d \tau .
$$

В дальнейшем зависимость некоторых параметров от $h$ будем опускать для краткости. Применяя формулу Коши для первых уравнений систем (1.1), (2.2) в виде

$$
w_{y}^{h}(t)=Y\left(t-\tau_{i}\right) w_{y}^{h}\left(\tau_{i}\right)+\int_{\tau_{i}}^{t} Y(t-\tau)\left(B v^{h}(\tau)+f_{0}(\tau)\right) d \tau
$$




$$
y(t)=Y\left(t-\tau_{i}\right) y\left(\tau_{i}\right)+\int_{\tau_{i}}^{t} Y(t-\tau)\left(B z(\tau)+f_{0}(\tau)\right) d \tau, \quad t \in\left[\tau_{i}, \vartheta\right]
$$

получаем

$$
\begin{gathered}
\exp \left(-\omega \tau_{i+1}\right)\left\|w_{y}^{h}\left(\tau_{i+1}\right)-y\left(\tau_{i+1}\right)\right\|_{n_{1}}^{2} \\
=\exp \left(-\omega \tau_{i+1}\right)\left\|Y(\delta)\left(w_{y}^{h}\left(\tau_{i}\right)-y\left(\tau_{i}\right)\right)+\int_{\tau_{i}}^{\tau_{i+1}} Y\left(\tau_{i+1}-\tau\right) B\left(v_{i}^{h}-z(\tau)\right) d \tau\right\|_{n_{1}}^{2} \\
=\exp \left(-\omega \tau_{i+1}\right)\left(\left\|Y(\delta)\left(w_{y}^{h}\left(\tau_{i}\right)-y\left(\tau_{i}\right)\right)\right\|_{n_{1}}^{2}+2\left(Y(\delta)\left(w_{y}^{h}\left(\tau_{i}\right)-y\left(\tau_{i}\right)\right), \int_{\tau_{i}}^{\tau_{i+1}} Y\left(\tau_{i+1}-\tau\right) B\left(v_{i}^{h}-z(\tau)\right) d \tau\right)_{n_{1}}\right. \\
\left.+\left\|\int_{\tau_{i}}^{\tau_{i+1}} Y\left(\tau_{i+1}-\tau\right) B\left(v_{i}^{h}-z(\tau)\right) d \tau\right\|_{n_{1}}^{2}\right) \leq \exp \left(-\omega \tau_{i+1}\right)\left\|Y(\delta)\left(w_{y}^{h}\left(\tau_{i}\right)-y\left(\tau_{i}\right)\right)\right\|_{n_{1}}^{2} \\
+2 \exp \left(-\omega \tau_{i+1}\right)\left(Y(\delta)\left(w_{y}^{h}\left(\tau_{i}\right)-y\left(\tau_{i}\right)\right), \int_{\tau_{i}}^{\tau_{i+1}} Y\left(\tau_{i+1}-\tau\right) B\left(v_{i}^{h}-z(\tau)\right) d \tau\right)_{n_{1}} \\
+\exp \left(-\omega \tau_{i+1}\right) \delta \int_{\tau_{i}}^{\tau_{i+1}}\left\|Y\left(\tau_{i+1}-\tau\right) B\left(v_{i}^{h}-z(\tau)\right)\right\|_{n_{1}}^{2} d \tau .
\end{gathered}
$$

Используя соотношение (1.2), первое неравенство из $(2.1)$ в виде $\|Y(\delta)\|_{n_{1}}^{2} \leq \exp (\omega \delta)$ и учитывая малость интегралов по отрезку $\left[\tau_{i}, \tau_{i+1}\right]$ от ограниченных функций, выводим оценки

$$
\begin{gathered}
\varepsilon_{h}\left(\tau_{i+1}\right) \leq \exp \left(-\omega \tau_{i}\right)\left\|w_{y}^{h}\left(\tau_{i}\right)-y\left(\tau_{i}\right)\right\|_{n_{1}}^{2}+\lambda_{i}+\mu_{i}+\alpha \int_{t_{0}}^{\tau_{i+1}}\left(\left\|v^{h}(\tau)\right\|_{n_{2}}^{2}-\|z(\tau)\|_{n_{2}}^{2}\right) d \tau \\
\left\|Y(\delta)\left(w_{y}^{h}\left(\tau_{i}\right)-y\left(\tau_{i}\right)\right)-Y(\delta)\left(w_{y}^{h}\left(\tau_{i}\right)-\xi\left(\tau_{i}\right)\right)\right\|_{n_{1}} \leq h \exp (\omega \delta / 2),
\end{gathered}
$$

где

$$
\begin{gathered}
\lambda_{i}=2 \exp \left(-\omega \tau_{i+1}\right)\left(Y(\delta)\left(w_{y}^{h}\left(\tau_{i}\right)-y\left(\tau_{i}\right)\right), \int_{\tau_{i}}^{\tau_{i+1}} Y\left(\tau_{i+1}-\tau\right) B\left(v_{i}^{h}-z(\tau)\right) d \tau\right)_{n_{1}} \\
\leq 2 \exp \left(-\omega \tau_{i+1}\right)\left(Y(\delta)\left(w_{y}^{h}\left(\tau_{i}\right)-\xi\left(\tau_{i}\right)\right), \int_{\tau_{i}}^{\tau_{i+1}} Y\left(\tau_{i+1}-\tau\right) B\left(v_{i}^{h}-z(\tau)\right) d \tau\right)_{n_{1}}+K_{1} \delta h \\
\mu_{i} \leq \exp \left(-\omega \tau_{i}\right) \delta \int_{\tau_{i}}^{\tau_{i+1}}\left\|B\left(v_{i}^{h}-z(\tau)\right)\right\|_{n_{1}}^{2} d \tau .
\end{gathered}
$$

Здесь и ниже через $K_{i}$ обозначаем вспомогательные положительные константы, которые могут быть выписаны явно. Далее, учитывая неравенство

$$
\left\|Y(\delta)\left(w_{y}^{h}\left(\tau_{i}\right)-\xi\left(\tau_{i}\right)\right)-\left(w_{y}^{h}\left(\tau_{i}\right)-\xi\left(\tau_{i}\right)\right)\right\|_{n_{1}} \leq K_{2} \delta\left\|w_{y}^{h}\left(\tau_{i}\right)-\xi\left(\tau_{i}\right)\right\|_{n_{1}},
$$

вытекающее из свойств фундаментальной матрицы, получаем для $\lambda_{i}$

$$
\left|\left(Y(\delta)\left(w_{y}^{h}\left(\tau_{i}\right)-\xi\left(\tau_{i}\right)\right), \int_{\tau_{i}}^{\tau_{i+1}} Y\left(\tau_{i+1}-\tau\right) B\left(v_{i}^{h}-z(\tau)\right) d \tau\right)_{n_{1}}-\left(\left(w_{y}^{h}\left(\tau_{i}\right)-\xi\left(\tau_{i}\right)\right), \int_{\tau_{i}}^{\tau_{i+1}} B\left(v_{i}^{h}-z(\tau)\right) d \tau\right)_{n_{1}}\right|
$$




$$
\begin{gathered}
\leq \mid\left(Y(\delta)\left(w_{y}^{h}\left(\tau_{i}\right)-\xi\left(\tau_{i}\right)\right), \int_{\tau_{i}}^{\tau_{i+1}} Y\left(\tau_{i+1}-\tau\right) B\left(v_{i}^{h}-z(\tau)\right) d \tau\right)_{n_{1}} \\
-\left(\left(w_{y}^{h}\left(\tau_{i}\right)-\xi\left(\tau_{i}\right)\right), \int_{\tau_{i}}^{\tau_{i+1}} Y\left(\tau_{i+1}-\tau\right) B\left(v_{i}^{h}-z(\tau)\right) d \tau\right)_{n_{1}} \mid \\
+\mid\left(\left(w_{y}^{h}\left(\tau_{i}\right)-\xi\left(\tau_{i}\right)\right), \int_{\tau_{i}}^{\tau_{i+1}} Y\left(\tau_{i+1}-\tau\right) B\left(v_{i}^{h}-z(\tau)\right) d \tau\right)_{n_{1}} \\
\quad-\left(\left(w_{y}^{h}\left(\tau_{i}\right)-\xi\left(\tau_{i}\right)\right), \int_{\tau_{i}}^{\tau_{i+1}} B\left(v_{i}^{h}-z(\tau)\right) d \tau\right)_{n_{1}} \mid \leq K_{3} \delta^{2} .
\end{gathered}
$$

Кроме того, $\mu_{i} \leq K_{4} \delta^{2}$. Используя (2.12), предыдущее неравенство и правило выбора модельного управления $v_{i}^{h}$ (см. (2.5), (2.6)), получим

$$
\begin{gathered}
\varepsilon_{h}\left(\tau_{i+1}\right) \leq \varepsilon_{h}\left(\tau_{i}\right)+\lambda_{i}+\mu_{i}+\alpha \int_{\tau_{i}}^{\tau_{i+1}}\left(\left\|v^{h}(\tau)\right\|_{n_{2}}^{2}-\|z(\tau)\|_{n_{2}}^{2}\right) d \tau \\
\leq \varepsilon_{h}\left(\tau_{i}\right)+2 \exp \left(-\omega \tau_{i+1}\right)\left(\left(w_{y}^{h}\left(\tau_{i}\right)-\xi\left(\tau_{i}\right)\right), \int_{\tau_{i}}^{\tau_{i+1}} B\left(v_{i}^{h}-z(\tau)\right) d \tau\right)_{n_{1}}+K_{1} \delta h+K_{3} \delta^{2}+K_{4} \delta^{2} \\
+\alpha \int_{\tau_{i}}^{\tau_{i+1}}\left(\left\|v^{h}(\tau)\right\|_{n_{2}}^{2}-\|z(\tau)\|_{n_{2}}^{2}\right) d \tau=\varepsilon_{h}\left(\tau_{i}\right)+\int_{\tau_{i}}^{\tau_{i+1}}\left(\left[2 \exp \left(-\omega \tau_{i+1}\right)\left(\left(w_{y}^{h}\left(\tau_{i}\right)-\xi\left(\tau_{i}\right)\right), B v_{i}^{h}\right)_{n_{1}}+\alpha\left\|v_{i}^{h}\right\|_{n_{2}}^{2}\right]\right. \\
\left.-\left[2 \exp \left(-\omega \tau_{i+1}\right)\left(\left(w_{y}^{h}\left(\tau_{i}\right)-\xi\left(\tau_{i}\right)\right), B z(\tau)\right)_{n_{1}}+\alpha\|z(\tau)\|_{n_{2}}^{2}\right]\right) d \tau+K_{1} \delta h+\left(K_{3}+K_{4}\right) \delta^{2} \\
\leq \varepsilon_{h}\left(\tau_{i}\right)+K_{5} \delta(h+\delta), \quad i \in[0:(l-1)] .
\end{gathered}
$$

Отсюда для любого $i \in[0:(l-1)]$ выводим, учитывая, что $\varepsilon_{h}\left(\tau_{0}\right)=0$ :

$$
\varepsilon_{h}\left(\tau_{i+1}\right) \leq K_{6}(h+\delta) ;
$$

это влечет оценку

$$
\left\|w_{y}^{h}\left(\tau_{i+1}\right)-y\left(\tau_{i+1}\right)\right\|_{n_{1}}^{2} \leq K_{7}(h+\delta+\alpha), \quad i \in[0:(l-1)] .
$$

В таком случае через формулу Коши имеем

$$
\begin{gathered}
\sup _{t \in T}\left\|\int_{t_{0}}^{t} Y(t-\tau) B\left(v^{h}(\tau)-z(\tau)\right) d \tau\right\|_{n_{1}}^{2} \leq K_{8}(h+\delta+\alpha), \\
\sup _{t \in T}\left\|\int_{t_{0}}^{t} Y(t-\tau) B\left(v^{h}(\tau)-z(\tau)\right) d \tau\right\|_{n_{1}} \leq K_{9}(h+\delta+\alpha)^{1 / 2} .
\end{gathered}
$$

Интегрируя по частям, получаем

$$
\int_{t_{0}}^{t} Y(t-\tau) B\left(v^{h}(\tau)-z(\tau)\right) d \tau=\left.Y(t-\tau) B \int_{t_{0}}^{\tau}\left(v^{h}(s)-z(s)\right) d s\right|_{t_{0}} ^{t}
$$




$$
\begin{gathered}
-\int_{t_{0}}^{t} \dot{Y}_{\tau}(t-\tau) B \int_{t_{0}}^{\tau}\left(v^{h}(s)-z(s)\right) d s d \tau=B \int_{t_{0}}^{t}\left(v^{h}(s)-z(s)\right) d s \\
\quad+\int_{t_{0}}^{t} A(t-\tau) Y(t-\tau) B \int_{t_{0}}^{\tau}\left(v^{h}(s)-z(s)\right) d s d \tau,
\end{gathered}
$$

откуда

$$
\begin{gathered}
\left\|B \int_{t_{0}}^{t}\left(v^{h}(s)-z(s)\right) d s\right\|_{n_{1}} \leq K_{9}(h+\delta+\alpha)^{1 / 2}+\left\|\int_{t_{0}}^{t} A(t-\tau) Y(t-\tau) B \int_{t_{0}}^{\tau}\left(v^{h}(s)-z(s)\right) d s d \tau\right\|_{n_{1}} \\
\leq K_{9}(h+\delta+\alpha)^{1 / 2}+\int_{t_{0}}^{t}\|A(t-\tau) Y(t-\tau)\|_{n_{1}}\left\|B \int_{t_{0}}^{\tau}\left(v^{h}(s)-z(s)\right) d s\right\|_{n_{1}} d \tau .
\end{gathered}
$$

Используя лемму Гронуолла и условие 1 , имеем

$$
\begin{aligned}
& d_{*}\left\|\int_{t_{0}}^{t}\left(v^{h}(s)-z(s)\right) d s\right\|_{n_{2}} \leq\left\|B \int_{t_{0}}^{t}\left(v^{h}(s)-z(s)\right) d s\right\|_{n_{1}} \\
& \leq K_{9}(h+\delta+\alpha)^{1 / 2} \exp \left(\int_{t_{0}}^{t}\|A(t-\tau) Y(t-\tau)\|_{n_{1}} d \tau\right) .
\end{aligned}
$$

Таким образом,

$$
\sup _{t \in T}\left\|\int_{t_{0}}^{t}\left(v^{h}(\tau)-z(\tau)\right) d \tau\right\|_{n_{2}} \leq K_{10}(h+\delta+\alpha)^{1 / 2} .
$$

Рассматривая (2.13) при $i=l-1$, получаем, отбрасывая из левой части неравенства положительное слагаемое,

$$
\left\|v^{h}(\cdot)\right\|_{L_{2}\left(T ; \mathbb{R}^{\left.n_{2}\right)}\right.}^{2} \leq\|z(\cdot)\|_{L_{2}\left(T ; \mathbb{R}^{\left.n_{2}\right)}\right.}^{2}+K_{11}(h+\delta) \alpha^{-1} .
$$

Далее, из (2.15), (2.16), применяя лемму об оценке интеграла от скалярного произведения с учетом ограниченности вариации абсолютно непрерывной функции $z(\cdot)$ (см. [3, с. 54]), выводим

$$
\begin{gathered}
\left\|v^{h}(\cdot)-z(\cdot)\right\|_{L_{2}\left(T ; \mathbb{R}^{n_{2}}\right)}^{2}=\left\|v^{h}(\cdot)\right\|_{L_{2}\left(T ; \mathbb{R}^{n_{2}}\right)}^{2}-2 \int_{t_{0}}^{\vartheta}\left(v^{h}(\tau), z(\tau)\right)_{n_{2}} d \tau+\|z(\cdot)\|_{L_{2}\left(T ; \mathbb{R}^{n_{2}}\right)}^{2} \\
\leq 2\|z(\cdot)\|_{L_{2}\left(T ; \mathbb{R}^{n_{2}}\right)}^{2}-2 \int_{t_{0}}^{\vartheta}\left(v^{h}(\tau), z(\tau)\right)_{n_{2}} d \tau+K_{11}(h+\delta) \alpha^{-1} \\
=2 \int_{t_{0}}^{\vartheta}\left(z(\tau)-v^{h}(\tau), z(\tau)\right)_{n_{2}} d \tau+K_{11}(h+\delta) \alpha^{-1} \leq K_{12}(h+\delta+\alpha)^{1 / 2}+K_{11}(h+\delta) \alpha^{-1}=K_{13} \rho(h),
\end{gathered}
$$

что доказывает оценку (2.11). Легко проверить, что она имеет порядок малости $O\left(h^{1 / 3}\right)$. 
Перейдем к доказательству основного утверждения (2.10). Введем второй функционал типа Ляпунова и оценим его изменение:

$$
\hat{\varepsilon}_{h}(t)=\exp (-\hat{\omega} t)\left\|w_{z}^{h}(t)-z(t)\right\|_{n_{2}}^{2}+\hat{\alpha}(h) \int_{t_{0}}^{t}\left(\left\|u^{h}(\tau)\right\|_{m}^{2}-\|u(\tau)\|_{m}^{2}\right) d \tau
$$

Применяя формулу Коши для вторых уравнений систем (1.1), (2.2) в виде

$$
\begin{gathered}
w_{z}^{h}(t)=Z\left(t-\tau_{i}\right) w_{z}^{h}\left(\tau_{i}\right)+\int_{\tau_{i}}^{t} Z(t-\tau)\left(D w_{y}^{h}(\tau)+E u^{h}(\tau)+f_{1}(\tau)\right) d \tau, \\
z(t)=Z\left(t-\tau_{i}\right) z\left(\tau_{i}\right)+\int_{\tau_{i}}^{t} Z(t-\tau)\left(D y(\tau)+E u(\tau)+f_{1}(\tau)\right) d \tau, \quad t \in\left[\tau_{i}, \vartheta\right],
\end{gathered}
$$

имеем

$$
\begin{gathered}
\exp \left(-\hat{\omega} \tau_{i+1}\right)\left\|w_{z}^{h}\left(\tau_{i+1}\right)-z\left(\tau_{i+1}\right)\right\|_{n_{2}}^{2} \\
=\exp \left(-\hat{\omega} \tau_{i+1}\right)\left\|Z(\delta)\left(w_{z}^{h}\left(\tau_{i}\right)-z\left(\tau_{i}\right)\right)+\int_{\tau_{i}}^{\tau_{i+1}} Z\left(\tau_{i+1}-\tau\right)\left(D\left(w_{y}^{h}(\tau)-y(\tau)\right)+E\left(u^{h}(\tau)-u(\tau)\right)\right) d \tau\right\|_{n_{2}}^{2} \\
=\exp \left(-\hat{\omega} \tau_{i+1}\right)\left(\left\|Z(\delta)\left(w_{z}^{h}\left(\tau_{i}\right)-z\left(\tau_{i}\right)\right)\right\|_{n_{2}}^{2}\right. \\
+2\left(Z(\delta)\left(w_{z}^{h}\left(\tau_{i}\right)-z\left(\tau_{i}\right)\right), \int_{\tau_{i}}^{\tau_{i+1}} Z\left(\tau_{i+1}-\tau\right)\left(D\left(w_{y}^{h}(\tau)-y(\tau)\right)+E\left(u^{h}(\tau)-u(\tau)\right)\right) d \tau\right)_{n_{2}} \\
\left.+\left\|\int_{\tau_{i}}^{\tau_{i+1}} Z\left(\tau_{i+1}-\tau\right)\left(D\left(w_{y}^{h}(\tau)-y(\tau)\right)+E\left(u^{h}(\tau)-u(\tau)\right)\right) d \tau\right\|_{n_{2}}^{2}\right) \\
+2 \exp \left(-\hat{\omega} \tau_{i+1}\right)\left(Z(\delta)\left(w_{z}^{h}\left(\tau_{i}\right)-z\left(\tau_{i}\right)\right), \int_{\tau_{i}}^{\tau_{i+1}} Z\left(\tau_{i+1}-\tau\right)\left(D\left(w_{y}^{h}(\tau)-y(\tau)\right)+E\left(u^{h}(\tau)-u(\tau)\right)\right) d \tau\right)_{n_{2}} \\
+2 \exp \left(-\hat{\omega} \tau_{i+1}\right) \delta \int_{\tau_{i}}^{\tau_{i+1}}\left\|Z\left(\tau_{i+1}-\tau\right)\right\|_{n_{2}}^{2}\left(\left\|D\left(w_{y}^{h}(\tau)-y(\tau)\right)\right\|_{n_{2}}^{2}+\left\|E\left(u^{h}(\tau)-u(\tau)\right)\right\|_{n_{2}}^{2}\right) d \tau .
\end{gathered}
$$

Используя второе неравенство из $(2.1)$ в виде $\|Z(\delta)\|_{n_{2}}^{2} \leq \exp (\hat{\omega} \delta)$ и учитывая малость интегралов по отрезку $\left[\tau_{i}, \tau_{i+1}\right]$ от ограниченных функций, выводим оценки

$$
\begin{gathered}
\hat{\varepsilon}_{h}\left(\tau_{i+1}\right) \leq \exp \left(-\hat{\omega} \tau_{i}\right)\left\|w_{z}^{h}\left(\tau_{i}\right)-z\left(\tau_{i}\right)\right\|_{n_{2}}^{2}+\hat{\lambda}_{i}+\hat{\mu}_{i}+\hat{\alpha} \int_{t_{0}}^{\tau_{i+1}}\left(\left\|u^{h}(\tau)\right\|_{m}^{2}-\|u(\tau)\|_{m}^{2}\right) d \tau \\
\left\|Z(\delta)\left(w_{z}^{h}\left(\tau_{i}\right)-z\left(\tau_{i}\right)\right)-Z(\delta)\left(w_{z}^{h}\left(\tau_{i}\right)-v^{h}\left(\tau_{i}\right)\right)\right\|_{n_{2}} \leq \exp (\hat{\omega} \delta / 2)\left\|v^{h}\left(\tau_{i}\right)-z\left(\tau_{i}\right)\right\|_{n_{2}},
\end{gathered}
$$

где

$$
\hat{\lambda}_{i}=2 \exp \left(-\hat{\omega} \tau_{i+1}\right)\left(Z(\delta)\left(w_{z}^{h}\left(\tau_{i}\right)-z\left(\tau_{i}\right)\right), \int_{\tau_{i}}^{\tau_{i+1}} Z\left(\tau_{i+1}-\tau\right)\left(D\left(w_{y}^{h}(\tau)-y(\tau)\right)+E\left(u^{h}(\tau)-u(\tau)\right)\right) d \tau\right)_{n_{2}}
$$




$$
\begin{gathered}
\leq 2 \exp \left(-\hat{\omega} \tau_{i+1}\right)\left(Z(\delta)\left(w_{z}^{h}\left(\tau_{i}\right)-v^{h}\left(\tau_{i}\right)\right), \int_{\tau_{i}}^{\tau_{i+1}} Z\left(\tau_{i+1}-\tau\right)\left(D\left(w_{y}^{h}(\tau)-y(\tau)\right)+E\left(u^{h}(\tau)-u(\tau)\right)\right) d \tau\right)_{n_{2}} \\
+\hat{K}_{1} \delta\left\|v^{h}\left(\tau_{i}\right)-z\left(\tau_{i}\right)\right\|_{n_{2}} \\
\hat{\mu}_{i} \leq 2 \exp \left(-\hat{\omega} \tau_{i}\right) \delta \int_{\tau_{i}}^{\tau_{i+1}}\left(\left\|D\left(w_{y}^{h}(\tau)-y(\tau)\right)\right\|_{n_{2}}^{2}+\left\|E\left(u^{h}(\tau)-u(\tau)\right)\right\|_{n_{2}}^{2}\right) d \tau .
\end{gathered}
$$

Здесь и ниже через $\hat{K}_{i}$ обозначаем вспомогательные положительные константы, которые могут быть выписаны явно. Далее, учитывая неравенство

$$
\left\|Z(\delta)\left(w_{z}^{h}\left(\tau_{i}\right)-v^{h}\left(\tau_{i}\right)\right)-\left(w_{z}^{h}\left(\tau_{i}\right)-v^{h}\left(\tau_{i}\right)\right)\right\|_{n_{2}} \leq \hat{K}_{2} \delta\left\|w_{z}^{h}\left(\tau_{i}\right)-v^{h}\left(\tau_{i}\right)\right\|_{n_{2}},
$$

вытекающее из свойств фундаментальной матрицы, получаем для $\hat{\lambda}_{i}$

$$
\begin{aligned}
& \mid\left(Z(\delta)\left(w_{z}^{h}\left(\tau_{i}\right)-v^{h}\left(\tau_{i}\right)\right), \int_{\tau_{i}}^{\tau_{i+1}} Z\left(\tau_{i+1}-\tau\right)\left(D\left(w_{y}^{h}(\tau)-y(\tau)\right)+E\left(u^{h}(\tau)-u(\tau)\right)\right) d \tau\right)_{n_{2}} \\
& \quad-\left(\left(w_{z}^{h}\left(\tau_{i}\right)-v^{h}\left(\tau_{i}\right)\right), \int_{\tau_{i}}^{\tau_{i+1}}\left(D\left(w_{y}^{h}(\tau)-y(\tau)\right)+E\left(u^{h}(\tau)-u(\tau)\right)\right) d \tau\right)_{n_{2}} \mid \\
& \leq \mid\left(Z(\delta)\left(w_{z}^{h}\left(\tau_{i}\right)-v^{h}\left(\tau_{i}\right)\right), \int_{\tau_{i}}^{\tau_{i+1}} Z\left(\tau_{i+1}-\tau\right)\left(D\left(w_{y}^{h}(\tau)-y(\tau)\right)+E\left(u^{h}(\tau)-u(\tau)\right)\right) d \tau\right)_{n_{2}} \\
& -\left(\left(w_{z}^{h}\left(\tau_{i}\right)-v^{h}\left(\tau_{i}\right)\right), \int_{\tau_{i}}^{\tau_{i+1}} Z\left(\tau_{i+1}-\tau\right)\left(D\left(w_{y}^{h}(\tau)-y(\tau)\right)+E\left(u^{h}(\tau)-u(\tau)\right)\right) d \tau\right)_{n_{2}} \mid \\
& +\mid\left(\left(w_{z}^{h}\left(\tau_{i}\right)-v^{h}\left(\tau_{i}\right)\right), \int_{\tau_{i}}^{\tau_{i+1}} Z\left(\tau_{i+1}-\tau\right)\left(D\left(w_{y}^{h}(\tau)-y(\tau)\right)+E\left(u^{h}(\tau)-u(\tau)\right)\right) d \tau\right)_{n_{2}} \\
& \quad-\left(\left(w_{z}^{h}\left(\tau_{i}\right)-v^{h}\left(\tau_{i}\right)\right), \int_{\tau_{i}}^{\tau_{i+1}}\left(D\left(w_{y}^{h}(\tau)-y(\tau)\right)+E\left(u^{h}(\tau)-u(\tau)\right)\right) d \tau\right)_{n_{2}} \mid \leq \hat{K}_{3} \delta^{2} .
\end{aligned}
$$

Кроме того, $\hat{\mu}_{i} \leq \hat{K}_{4} \delta^{2}$. Используя (2.14), (2.17), предыдущее неравенство и правило выбора модельного управления $u_{i}^{h}$ (см. $\left.(2.7),(2.8)\right)$, получим

$$
\begin{gathered}
\hat{\varepsilon}_{h}\left(\tau_{i+1}\right) \leq \hat{\varepsilon}_{h}\left(\tau_{i}\right)+\hat{\lambda}_{i}+\hat{\mu}_{i}+\hat{\alpha} \int_{\tau_{i}}^{\tau_{i+1}}\left(\left\|u^{h}(\tau)\right\|_{m}^{2}-\|u(\tau)\|_{m}^{2}\right) d \tau \\
\leq \hat{\varepsilon}_{h}\left(\tau_{i}\right)+2 \exp \left(-\hat{\omega} \tau_{i+1}\right)\left(\left(w_{z}^{h}\left(\tau_{i}\right)-v^{h}\left(\tau_{i}\right)\right), \int_{\tau_{i}}^{\tau_{i+1}}\left(D\left(w_{y}^{h}(\tau)-y(\tau)\right)+E\left(u^{h}(\tau)-u(\tau)\right)\right) d \tau\right)_{n_{2}} \\
+\hat{K}_{1} \delta\left\|v^{h}\left(\tau_{i}\right)-z\left(\tau_{i}\right)\right\|_{n_{2}}+\hat{K}_{3} \delta^{2}+\hat{K}_{4} \delta^{2}+\hat{\alpha} \int_{\tau_{i}}^{\tau_{i+1}}\left(\left\|u^{h}(\tau)\right\|_{m}^{2}-\|u(\tau)\|_{m}^{2}\right) d \tau
\end{gathered}
$$




$$
\begin{gathered}
=\hat{\varepsilon}_{h}\left(\tau_{i}\right)+\int_{\tau_{i}}^{\tau_{i+1}}\left(\left[2 \exp \left(-\hat{\omega} \tau_{i+1}\right)\left(\left(w_{z}^{h}\left(\tau_{i}\right)-v^{h}\left(\tau_{i}\right)\right), E u_{i}^{h}\right)_{n_{2}}+\hat{\alpha}\left\|u_{i}^{h}\right\|_{m}^{2}\right]\right. \\
\left.-\left[2 \exp \left(-\hat{\omega} \tau_{i+1}\right)\left(\left(w_{z}^{h}\left(\tau_{i}\right)-v^{h}\left(\tau_{i}\right)\right), E u(\tau)\right)_{n_{2}}+\hat{\alpha}\|u(\tau)\|_{m}^{2}\right]\right) d \tau \\
\quad+\hat{K}_{1} \delta\left\|v^{h}\left(\tau_{i}\right)-z\left(\tau_{i}\right)\right\|_{n_{2}}+\left(\hat{K}_{3}+\hat{K}_{4}\right) \delta^{2}+\hat{K}_{5} \delta(h+\delta+\alpha)^{1 / 2} \\
\leq \hat{\varepsilon}_{h}\left(\tau_{i}\right)+\hat{K}_{1} \delta\left\|v^{h}\left(\tau_{i}\right)-z\left(\tau_{i}\right)\right\|_{n_{2}}+\left(\hat{K}_{3}+\hat{K}_{4}\right) \delta^{2}+\hat{K}_{5} \delta(h+\delta+\alpha)^{1 / 2}, \quad i \in[0:(l-1)] .
\end{gathered}
$$

Отсюда для любого $i \in[0:(l-1)]$ выводим, учитывая, что $\hat{\varepsilon}_{h}\left(\tau_{0}\right)=0$ :

$$
\hat{\varepsilon}_{h}\left(\tau_{i+1}\right) \leq \hat{K}_{1} \delta \sum_{i=1}^{l-1}\left\|v^{h}\left(\tau_{i}\right)-z\left(\tau_{i}\right)\right\|_{n_{2}}+\hat{K}_{6}\left(\delta+(h+\delta+\alpha)^{1 / 2}\right) .
$$

Учитывая (2.11), оценим

$$
\int_{\tau_{0}}^{\vartheta}\left\|v^{h}(\tau)-z(\tau)\right\|_{n_{2}} d \tau \leq \hat{K}_{7}\left\|v^{h}(\cdot)-z(\cdot)\right\|_{L_{2}\left(T ; \mathbb{R}^{n_{2}}\right)} \leq \hat{K}_{8}(\rho(h))^{1 / 2},
$$

что через определение интегральной суммы влечет $\hat{K}_{1} \delta \sum_{i=1}^{l-1}\left\|v^{h}\left(\tau_{i}\right)-z\left(\tau_{i}\right)\right\|_{n_{2}} \leq \hat{K}_{9}(\rho(h))^{1 / 2}$. Таким образом,

$$
\hat{\varepsilon}_{h}\left(\tau_{i+1}\right) \leq \hat{K}_{9}(\rho(h))^{1 / 2}+\hat{K}_{6}\left(\delta+(h+\delta+\alpha)^{1 / 2}\right) \leq \hat{K}_{10}(\rho(h))^{1 / 2},
$$

откуда следует оценка $\left\|w_{z}^{h}\left(\tau_{i+1}\right)-z\left(\tau_{i+1}\right)\right\|_{n_{2}}^{2} \leq \hat{K}_{11}\left((\rho(h))^{1 / 2}+\hat{\alpha}\right), i \in[0:(l-1)]$. В таком случае, используя формулу Коши и соотношение (2.14), имеем

$$
\begin{gathered}
\sup _{t \in T}\left\|\int_{t_{0}}^{t} Z(t-\tau)\left(D\left(w_{y}^{h}(\tau)-y(\tau)\right)+E\left(u^{h}(\tau)-u(\tau)\right)\right) d \tau\right\|_{n_{2}}^{2} \leq \hat{K}_{12}\left((\rho(h))^{1 / 2}+\hat{\alpha}\right), \\
\sup _{t \in T}\left\|\int_{t_{0}}^{t} Z(t-\tau) E\left(u^{h}(\tau)-u(\tau)\right) d \tau\right\|_{n_{2}} \leq \hat{K}_{13}\left((\rho(h))^{1 / 2}+\hat{\alpha}\right)^{1 / 2}+\hat{K}_{14}(h+\delta+\alpha)^{1 / 2} \\
\leq \hat{K}_{15}\left((\rho(h))^{1 / 2}+\hat{\alpha}\right)^{1 / 2} .
\end{gathered}
$$

Действуя в полном соответствии с выводом оценки (2.15), т. е. интегрируя по частям, применяя лемму Гронуолла и условие 2 , получаем

$$
\sup _{t \in T}\left\|\int_{t_{0}}^{t}\left(u^{h}(\tau)-u(\tau)\right) d \tau\right\|_{m} \leq \hat{K}_{16}\left((\rho(h))^{1 / 2}+\hat{\alpha}\right)^{1 / 2} .
$$

Рассматривая (2.18) при $i=l-1$, получаем, отбрасывая из левой части неравенства положительное слагаемое,

$$
\left\|u^{h}(\cdot)\right\|_{L_{2}\left(T ; \mathbb{R}^{m}\right)}^{2} \leq\|u(\cdot)\|_{L_{2}\left(T ; \mathbb{R}^{m}\right)}^{2}+\hat{K}_{17}(\rho(h))^{1 / 2} \hat{\alpha}^{-1} .
$$

Далее, по аналогии с выкладками при выводе (2.11) из первой части доказательства, из (2.19), (2.20), применяя лемму об оценке интеграла от скалярного произведения с учетом ограниченности вариации функции $u(\cdot)$ (см. [3, с. 54]), имеем

$$
\left\|u^{h}(\cdot)-u(\cdot)\right\|_{L_{2}\left(T ; \mathbb{R}^{m}\right)}^{2}=\left\|u^{h}(\cdot)\right\|_{L_{2}\left(T ; \mathbb{R}^{m}\right)}^{2}-2 \int_{t_{0}}^{\vartheta}\left(u^{h}(\tau), u(\tau)\right)_{m} d \tau+\|u(\cdot)\|_{L_{2}\left(T ; \mathbb{R}^{m}\right)}^{2}
$$




$$
\begin{gathered}
\leq 2\|u(\cdot)\|_{L_{2}\left(T ; \mathbb{R}^{m}\right)}^{2}-2 \int_{t_{0}}^{\vartheta}\left(u^{h}(\tau), u(\tau)\right)_{m} d \tau+\hat{K}_{17}(\rho(h))^{1 / 2} \hat{\alpha}^{-1} \\
=2 \int_{t_{0}}^{\vartheta}\left(u(\tau)-u^{h}(\tau), u(\tau)\right)_{m} d \tau+\hat{K}_{17}(\rho(h))^{1 / 2} \hat{\alpha}^{-1} \\
\leq \hat{K}_{18}\left((\rho(h))^{1 / 2}+\hat{\alpha}\right)^{1 / 2}+\hat{K}_{17}(\rho(h))^{1 / 2} \hat{\alpha}^{-1}=\hat{K}_{19} \hat{\rho}(h),
\end{gathered}
$$

что доказывает (2.10) и, следовательно, теорему.

Полученная оценка, опирающаяся на условие 2 , фактически позволяет сделать вывод о единственности искомого возмущения. Легко проверить, что она имеет порядок малости $O\left(h^{1 / 18}\right)$. Отметим, что оптимальность полученных оценок по порядку не исследуется; важен сам факт сходимости последовательности модельных управлений к реальной функции при $h \rightarrow 0$.

\section{Заключение}

В работе рассмотрена задача восстановления неизвестного внешнего воздействия для системы линейных обыкновенных дифференциальных уравнений в постановке, предполагающей динамическую реконструкцию возмущения на основе неточной дискретной информации о части координат фазовой траектории. Новизна постановки состоит в том, что неизвестная функция, подлежащая восстановлению, стеснена геометрическими ограничениями и не входит в измеряемую компоненту. Разработан конечношаговый программно реализуемый алгоритм решения, основанный на методе вспомогательных позиционно управляемых моделей, получена оценка его точности.

В качестве одного из направлений развития тематики планируется исследовать задачу гарантированного управления динамической системой смешанного типа, содержащей стохастическую и детерминированную компоненты. Искомое управление детерминированной компонентой, обеспечивающее достижение заданного значения критерия качества независимо от реализации неизвестной помехи, следует находить в условиях дефицита информации, например в предположении о возможности измерения некоторого количества реализаций стохастической компоненты в дискретные моменты времени с последующей реконструкцией второй компоненты в соответствии с разработанной ранее методикой сведения, на базе метода моментов, обратной задачи для стохастического уравнения к задаче для системы ОДУ [10].

\section{СПИСОК ЛИТЕРАТУРЫ}

1. Кряжимский А.В., Осипов Ю.С. О моделировании управления в динамической системе // Изв. АН СССР. Техн. кибернетика. 1983. № 2. С. 51-60.

2. Osipov Yu.S., Kryazhimskii A.V. Inverse problems for ordinary differential equations: dynamical solutions. L.: Gordon and Breach, 1995. 625 p.

3. Максимов В.И. Задачи динамического восстановления входов бесконечномерных систем. Екатеринбург: Изд-во УрО РАН, 2000. 305 с.

4. Красовский Н.Н., Субботин А.И. Позиционные дифференциальные игры. М.: Наука, 1984. $456 \mathrm{c}$.

5. Тихонов А.Н., Арсенин В.Я. Методы решения некорректных задач. М.: Наука, 1978. 142 с.

6. Кряжимский А.В., Осипов Ю.С. Об устойчивом позиционном восстановлении управления по измерениям части координат // Некоторые задачи управления и устойчивости / ред. А. В.Ким, В. И. Максимов. Свердловск: Изд-во УрО АН СССР, 1989. С. 33-47.

7. Осипов Ю.С., Кряжимский А.В., Максимов В.И. Методы динамического восстановления входов управляемых систем. Екатеринбург: Изд-во УрО РАН, 2011. 291 с. 
8. Близорукова М.С., Максимов В.И. Об одной задаче управления при неполной информации // Автоматика и телемеханика. 2006. № 3. С. 131-142.

9. Maksimov V.I. On dynamical reconstruction of an input in a linear system under measuring a part of coordinates // J. Inverse Ill-Posed Problems. 2018. Vol. 26, no. 3. P. 395-410. doi: $10.1515 /$ jiip-2017-0118.

10. Розенберг В.Л. Динамическая реконструкция возмущений в квазилинейном стохастическом дифференциальном уравнении // Журн. вычисл. математики и мат. физики. 2018. Т. 58, № 7. C. 1121-1131. doi: 10.31857/S004446690001461-8 .

Поступила 16.01.2019

После доработки 5.02.2019

Розенберг Валерий Львович Принята к публикации 11.02.2019

канд. физ.-мат. наук, старший науч. сотрудник

Институт математики и механики им. Н. Н. Красовского УрО РАН;

Уральский федеральный университет

г. Екатеринбург

e-mail: rozen@imm.uran.ru

\section{REFERENCES}

1. Kryazhimskii A.V., Osipov Yu.S. Modelling of a control in a dynamic system. Engrg. Cybernetics, 1983, vol. 21, no. 2, pp. 38-47.

2. Osipov Yu.S., Kryazhimskii A.V. Inverse problems for ordinary differential equations: dynamical solutions. L.: Gordon and Breach, 1995, 625 p. ISBN: 2881249442.

3. Maksimov V.I. Zadachi dinamicheskogo vosstanovleniya vkhodov beskonechnomernykh sistem [Problems of dynamic restoration of the inputs of infinite-dimensional systems]. Ekaterinburg: Ross. Akad. Nauk Publ., 2000, 305 p. ISBN: 5-7691-1082-1.

4. Krasovskii N.N., Subbotin A.I. Game-theoretical control problems. N Y: Springer, 1988, 517 p. ISBN: $978-$ 1-4612-8318-8. Original Russian text published in Krasovskii N.N., Subbotin A.I. Pozitsionnye differentsial'nye igry, Moscow, Nauka Publ., 1984, 456 p.

5. Tikhonov A.N., Arsenin V.Ya. Methods for solutions of ill-posed problems. N Y: Wiley, 1977, 258 p. ISBN: 0470991240 . Original Russian text (2nd ed.) published in Tikhonov A.N., Arsenin V.Ya. Metody resheniya nekorrektnykh zadach, Moscow: Nauka Publ., 1979, 285 p.

6. Kryazhimskii A.V., Osipov Yu.S. Stable positional reconstruction of a control from measurements of some of the coordinates. In: A. V. Kim and V. I. Maksimov (eds.) Some control and stability problems, Sverdlovsk: Akad. Nauk SSSR Ural. Otdel. Publ., 1989, pp. 33-47, 108-109 (in Russian).

7. Osipov Yu. S., Kryazhimskii A.V., Maksimov V.I. Metody dinamicheskogo vosstanovleniya vkhodov upravlyaemykh sistem [Dynamic recovery methods for inputs of control systems]. Yekaterinburg: UrO RAN Publ., 2011, 291 p.

8. Blizorukova M.S., Maksimov V.I. A control problem with incomplete information. Autom. Remote Control, 2006, vol. 67, no. 3, pp. 461-471. doi: 10.1134/S0005117906030106.

9. Maksimov V.I. On dynamical reconstruction of an input in a linear system under measuring a part of coordinates. J. Inverse Ill-Posed Problems, 2018, vol. 26, no. 3, pp. 395-410. doi: 10.1515/jiip-2017-0118.

10. Rozenberg V. L. Dynamic reconstruction of disturbances in a quasilinear stochastic differential equation. Comput. Math. Math. Physics, 2018, vol. 58, no. 7, pp. 1071-1080. doi: 10.1134/S096554251807014X .

Received January 16, 2018

Revised February 5, 2019

Accepted February 11, 2019

Valeriy Lvovich Rozenberg, Cand. Sci. (Phys.-Math.), Krasovskii Institute of Mathematics and Mechanics of the Ural Branch of the Russian Academy of Sciences, Yekaterinburg, 620108 Russia; Ural Federal University, Yekaterinburg, 620002 Russia, e-mail: rozen@imm.uran.ru. 